\title{
Tình trạng nhiễm độc tố vi nấm trong thực phẩm tại một số tỉnh, thành phố dịa phương miển Bắc Việt Nam nằm 2019
}

\author{
Nguyễn Thị Hà Bình*, Nguyễn Ngọc Sơn, Nguyễn Thị Lan, Trần Cao Sơn \\ Viện Kiểm nghiệm an toàn vệ sinh thực phẩm Quốc gia
}

(Ngày đến tòa soạn: 04/6/2020; Ngày chấp nhận đăng: 28/8/2020)

\section{Tóm tắt}

Nghiên cứu đã thực hiện đánh giá hàm lượng độc tố vi nấm aflatoxin, ochratoxin $\mathrm{A}$, zearalenone, deoxynivalenol and fumonisin B1 trong một số sản phẩm thực phẩm bao gồm bánh kẹo, bột thính trộn, bột ngũ cốc dinh dưỡng, thực phẩm bảo vệ sức khỏe, ngũ cốc nguyên liệu và gia vị các loại. Mẫu thực phẩm được chiết và làm sạch bằng phương pháp QuEChERS, sau đó dịch chiết được phân tích bằng thiết bị sắc ký lỏng khối phổ hai lân (LC-MS/MS). Giới hạn phát hiện của phương pháp cho aflatoxin, fumonisin B1, ochratoxin A, deoxynivalenol, zearalenone lần lượt là 0,$5 ; 30 ; 0,5 ; 60 ; 3,0 \mu \mathrm{g} / \mathrm{kg}$. Độ thu hồi của phương pháp trong khoảng 70 - 110\%. Độ lệch chuẩn tương đối RSD trong khoảng 5,1 - 13\%. Kết quả khảo sát 300 mẫu thực phẩm các loại cho thấy có 43 mẫu nhiễm độc tố vi nấm chiếm tỷ lệ $14,3 \%$, chủ yếu tập trung vào nhóm ngũ cốc chưa qua chế biến. Trong đó phát hiện 10 mẫu gia vị, 04 mẫu nguyên liệu thực phẩm, 01 mẫu kẹo, 01 mẫu bột ngũ cốc dinh dưỡng, 01 mẫu thực phẩm bảo vệ sức khỏe phát hiện có nhiễm aflatoxin, ochratoxin A vượt ngưỡng tối đa cho phép của Bộ Y tế theo quy chuẩn QCVN 8-1:2011/BYT.

Tư khóa: LC-MS/MS, aflatoxin, ochratoxin A, deoxynivalenol, zearalenone, fumonisin B1.

\section{1. ĐĂT VẤN ĐỀ}

Độc tố vi nấm (mycotoxin) là các độc tố do nấm mốc Aspergillus, Penicillium và Fusarium sinh ra trong quá trình chuyển hoá dinh dưỡng từ ngũ cốc và các nguyên liệu khác [1-3]. Hiện nay có trên 400 loại độc tố vi nấm được phát hiện, trong đó các độc tố vi nấm chính gồm có: aflatoxin, ochratoxin, deoxynivalenol, zearalenone, patulin, fumonisin, citrinin,...

Nhiêu nghiên cứu cho thấy độc tố vi nấm có thể có mặt trong nhiều loại thực phẩm. Một khảo sát của Romani từ năm 2008 đến 2010 trên các mẫu ngô, lúa mì, lúa mạch, yến mạch phát hiện $71,6 \%$ nhiễm deoxynivalenol, 40\% nhiễm aflatoxin, 16\% nhiễm ochratoxin A, 32\% nhiễm zearalenone [6]. Tại Bỉ, theo một báo cáo năm 2009 phát hiện fumonisin và ochratoxin trong một số thực phẩm bổ sung [7]. Ở Autralia năm 2009 đã khảo sát sự có mặt của 19 loại độc tố vi nấm có thể có trong 87 loại thực phẩm đã bị để mốc bao gồm bánh ngọt, trái cây, pho mai, mứt, phát hiện 49 mẫu dương tính với các loại độc tố vi nấm. Một nghiên cứu khác của Autralia năm 2009 còn cho thấy nông độ mycotoxin đáng kể được tìm thấy trong phân không bị mốc của các mẫu được khảo sát. Do đó, người ta đã kết luận rằng việc tận dụng thực phẩm bằng cách loại bỏ phân bị mốc và tiêu thụ phần còn lại là không an toàn [8]. Ở Việt Nam, theo nghiên cứu của Đỗ Hữu Tuấn và cộng sự, ngô và lạc là các đối tượng thực phẩm có tỷ lệ nhiễm độc tố vi nấm cao nhất [9]. 
Cơ quan quốc tế nghiên cứu về ung thư (IARC) đã phân loại aflatoxin là chất gây ung thư cho người (nhóm 1), ochratoxin $A$ và fumonisin là chất có thể gây ung thư đối với người (nhóm 2B) và zearalenone là chất gây ung thư nhóm 3 [4]. Ở liều lượng cao, độc tố vi nấm có thể gây ra các triệu chứng ngộ độc cấp tính như tác động vào gan, thận, buông trứng, nếu nhiễm độc kéo dài có thể gây ung thư [4-5]. Vì các tác hại mà độc tố này gây ra cho người và động vật nên giới hạn cho phép của độc tố trong thực phẩm rất thấp. Mức tối đa cho phép $(\mathrm{ML})$ của các độc tố vi nấm đã được Bộ Y tế quy định trong QCVN 8-1 : 2011/BYT như sau: aflatoxin trong khoảng từ $0,1-15 \mu \mathrm{g} / \mathrm{kg}$, ochratoxin A trong khoảng từ $0,5-80 \mu \mathrm{g} / \mathrm{kg}$, deoxynivalenol trong khoảng từ $200-1.750 \mu \mathrm{g} / \mathrm{kg}$, zearalenone trong khoảng từ $20-400 \mu \mathrm{g} / \mathrm{kg}$, fumonisin trong khoảng từ $200-4.000 \mu \mathrm{g} / \mathrm{kg}$.

Trong nghiên cứu này, nhóm nghiên cứu đã tiến hành khảo sát 05 loại độc tố vi nấm gồm: aflatoxin, ochratoxin $\mathrm{A}$, fuminosin B1, deoxynivalenol, zearalenone trong 300 mẫu thực phẩm (bánh kẹo, bột thính trộn, bột ngũ cốc dinh dưỡng, thực phẩm bảo vệ sức khỏe, ngũ cốc nguyên liệu và gia vị các loại) để đánh giá tỷ lệ nhiễm độc tố vi nấm và mức độ nhiễm độc tố vi nấm trong các mẫu thực phẩm được lấy.

\section{VẠT LIỆU VÀ PHƯƠNG PHÁP NGHIÊN CƯU}

\section{1. Đối tượng nghiên cứu}

Aflatoxin B1, aflatoxin tổng số, ochratoxin A, deoxynivalenol, zearalenone, fumonisin B1 là các độc tố vi nấm được nghiên cứu. Đối tượng mẫu khảo sát được lấy trên thị trường tại các tỉnh Hà Nội, Bắc Giang, Hà Giang.

\subsection{Lấy mẫu}

Phương thức lấy mẫu được thực hiện theo Thông tư 14/2011/TT-BYT của Bộ Y tế về việc lấy mẫu thực phẩm phục vụ thanh tra, kiểm tra chất lượng vệ sinh an toàn thực phẩm. Mẫu được lấy ngẫu nhiên tại Hà Nội, Bắc Giang, Hà Giang. Tổng số mẫu là 300 mẫu (Bảng 1).

Bảng 1. Kế hoạch lấy mẫu

\begin{tabular}{cccc}
\hline Đối tượng mẫu & Hà Nội & Bắc Giang & Hà Giang \\
\hline Bánh & 20 & 10 & 10 \\
Bột thính trộn & 30 & 15 & 15 \\
Bột ngũ cốc dinh duỗng & 30 & 15 & 15 \\
Thụ̂c phẩm bảo vệ súc khỏe & 20 & 10 & 10 \\
Nguyên liệu thực phẩm & 30 & 15 & 10 \\
Gia vị các loại & 20 & 10 & 75 \\
Tổng số & $\mathbf{1 5 0}$ & $\mathbf{7 5}$ & \\
\hline
\end{tabular}

\subsection{Hóa chất, chất chuẩn}

Các chất chuẩn aflatoxin B1, aflatoxin B2, aflatoxin $\mathrm{G} 1$, aflatoxin $\mathrm{G} 2$, ochratoxin $\mathrm{A}$, deoxynivalenol, zearalenone, fumonisin B1 được mua từ hãng Sigma (Mỹ). Các hóa chất acetonitril, acid formic đặc là loại tinh khiết phân tích dùng cho sắc ký được cung cấp bởi Merck 
(Đức). Natri chloride, magie sulphate khan của hãng Merck (Đức). Bột làm sạch C18 của hãng Agilent (Mỹ).

\section{4. Điều kiện thiết bị}

Thiết bị chính được sử dụng là hệ thống sắc ký lỏng khối phổ hai lần Triple Quad 5500 của SCIEX (Mỹ). Ngoài ra, các thiết bị thông thường khác của phòng thí nghiệm gồm: cân phân tích độ chính xác 0,1 mg (MS-205DU, Mettler), máy lắc xoáy (Genius, IKA), máy ly tâm tốc độ tối đa 18.000 vòng/phút (Mikro 220R, Hettich).

Điều kiện phân tích LC-MS/MS như sau: cột Symmestry C18 (150 mm × 0,46 mm × 3,5 $\mu \mathrm{m})$. Thể tích tiêm mẫu: $10 \mu \mathrm{L}$. Chương trình gradient pha động gồm 2 kênh $\mathrm{HCOOH} 0,1 \%$ trong nước $(\mathrm{A})$ và acetonitril $(\mathrm{B})$. Ban đầu giữ kênh $\mathrm{A}$ ở $80 \%$ trong 2 phút, sau đó giảm xuống $10 \%$ trong 4 phút, giữ ổn định trong 3 phút, tăng lên $80 \%$ trong 1 phút, giữ ổn định trong 5 phút. Tổng thời gian phân tích trên LC-MS/MS là 15 phút.

Các thông số tối ưu của điều kiện khối phổ (ESI+/-): điện thế nguồn (IS) $5000 \mathrm{~V} /-4000$ $\mathrm{V}$, nhiệt độ nguồn (TEM) $450^{\circ} \mathrm{C}$, khí nguôn 1 (GS1) 25 psi, khí nguôn 2 (GS2) 20 psi, khí màng (CUR) 20 psi, thế tạo hạt $(\mathrm{DP}) 130 \mathrm{~V}$, thế đâuu vào $(\mathrm{EP}) 9 \mathrm{~V}$ và khí phân mảnh $(\mathrm{CAD}) 7$ psi. Các thông số mảnh mẹ, mảnh con được trình bày tại Bảng 2.

Bảng 2. Các điêu kiện phân tích độc tố vi nấm bằng ESI(士)-MS/MS

\begin{tabular}{cccc}
\hline Chất phân tích & Mảnh $\boldsymbol{m e ̣ ( m / z )}$ & Mảnh con $(\boldsymbol{m} / \boldsymbol{z})$ & Năng lự̛ng va chạm $(\mathbf{C E}, \boldsymbol{e V})$ \\
\hline Aflatoxin B1 & 313 & $241^{*} / 269$ & $41 / 37$ \\
Aflatoxin B2 & 315 & $259^{*} / 287$ & $35 / 43$ \\
AflatoxinG1 & 329 & $243^{*} / 215$ & $39 / 33$ \\
Aflatoxin G2 & 331 & $245^{*} / 285$ & $47 / 41$ \\
Fumonisin B1 & 722 & $352^{*} / 334$ & $45 / 49$ \\
Fumonisin B2 & 706,9 & $336^{*} / 318$ & $47 / 47$ \\
Zearalenone & 317 & $175^{*} / 131$ & $-25 /-25$ \\
Deoxynivalenl & 295 & $265^{*} / 138$ & $-14 /-20$ \\
Ochratoxin A & 402 & $358^{*} / 167$ & $-24 /-35$ \\
\hline
\end{tabular}

Ghi chú: ${ }^{\star}$ Mảnh định lượng

\subsection{Xử lý mẫu}

Mẫu được phân tích bằng phương pháp QuEChERS, phương pháp xây dựng nội bộ đã được công nhận bởi Văn phòng công nhận chất lượng BOA. Cân chính xác khoảng $5 \mathrm{~g}$ mẫu vào ống ly tâm $50 \mathrm{~mL}$. Thêm $10 \mathrm{~mL}$ nước cất, lắc đều để nước thấm hoàn toàn vào mẫu. Thêm $5 \mathrm{~mL}$ dung môi chiết acetonitril chứa $1 \%$ acid formic, lắc trên máy lắc ngang tại tốc độ 140 vòng/phút trong 30 phút. Thêm hỗn hợp muối chiết gồm $2,0 \pm 0,05 \mathrm{~g} \mathrm{MgSO}_{4}$ và $0,5 \pm 0,01 \mathrm{~g} \mathrm{NaCl}$, lắc bằng tay trong 1 phút để muối hoàn toàn đồng nhất với dịch mẫu và ly tâm ở 6.000 vòng/phút trong 5 phút. Hút $1 \mathrm{~mL}$ dịch chiết vào ống chiết pha rắn $\mathrm{d}-\mathrm{SPE}$ chứa $0,1 \pm 0,05 \mathrm{~g} \mathrm{C} 18$ và $0,15 \pm 0,02$ $\mathrm{g} \mathrm{MgSO}_{4}$. Lắc xoáy trong 1 phút bằng máy lắc xoáy. Ly tâm tại 13.000 vòng/phút trong 2 phút bằng máy ly tâm. Lấy dịch ly tâm vào lọ đựng mẫu $2 \mathrm{~mL}$ và phân tích trên hệ thống LC-MS/MS. 


\section{KẾT QUẢ VÀ BÀN LUẬN}

\subsection{Thẩm định phương pháp}

Trước khi tiến hành phân tích mẫu thực, phương pháp phân tích đã được thẩm định tối ủu các thông số: độ chọn lọc, khoảng tuyến tính, $\mathrm{LOD} / \mathrm{LOQ}$, độ thu hồi và độ chụm. $\mathrm{LOD}$ của các chất được xác định bằng cách thêm chuẩn vào mẫu trắng ở mức LOQ ước lượng và tiến hành phân tích từ ban đầu. Xác định tỷ lệ tín hiệu của chuẩn trên tín hiệu nhiễu. LOD là nông độ chất phân tích tại mức $S / N \geq 3$. Kết quả LOD chi tiết của các chất được trình bày ở Bảng 3 . Giới hạn định lượng của phương pháp cho các mycotoxin đều thấp hơn mức giới hạn tối đa cho phép theo Quy định hiện hành của Châu Âu cho các đối tượng thực phẩm (Ủy ban Châu Âu, 2006a; Châu Âu Ủy ban, 2010).

Bảng 3. Giới hạn phát hiện và giới hạn định lượng của các độc tố vi nấm

\begin{tabular}{cccccc}
\hline Thôngsố & Aflatoxin & Ochratoxin A & Fumonisin B1 & Zearalenone & Deoxynivalenol \\
\hline $\boldsymbol{L O D}(\boldsymbol{\mu g} / \mathbf{k g})$ & 0,5 & 0,5 & 30 & 3,0 & 60 \\
$\boldsymbol{L O Q}(\boldsymbol{\mu} \boldsymbol{g} / \mathbf{k g})$ & 1,5 & 1,5 & 100 & 10 & 200 \\
\hline
\end{tabular}

Độ thu hôi và độ chụm của các phương pháp được đánh giá ở ba mức nồng độ khác nhau (LOQ, ML, 2ML) cho các loại nền mẫu thực phẩm gồm bột ngũ cốc, bánh kẹo, thực phẩm bảo vệ sức khỏe, ớt bột. Các kết quả của các thí nghiệm được được thể hiện ở Bảng 4.

Bảng 4. Độ thu hồi và độ chụm của các độc tố vi nấm trong thực phẩm

\begin{tabular}{ccc}
\hline Thông số & Độ thu hồi $(\mathrm{R} \%)$ & Độ chụm (RSD \%) \\
\hline Aflatoxin & $70-110$ & $9,1-13$ \\
Ochratoxin A & $75-108$ & $8,0-12$ \\
Deoxynivalenol & $92-105$ & $5,1-9,0$ \\
Zearalenone & $80-103$ & $10-13$ \\
Fumonisin B1 & $82-105$ & $5,5-8,2$ \\
\hline
\end{tabular}

Aflatoxin B1, aflatoxin tổng số, fumonisin B1 được phân tích ở chế độ ion hóa tia điện ESI dương. Ochratoxin A, deoxynivalenol, zearalenone được phân tích ở chế độ ESI âm.

Các kết quả này đáp ứng các tiêu chí theo Quy định EC số 401/2006 của Ủy ban châu Âu về thẩm định phương pháp phân tích. Do đó, phương pháp phân tích đủ tin cậy và độ nhạy phân tích các độc tố vi nấm.

\subsection{Kết quả phân tích mẫu thực tế}

Mẫu được lấy tại 03 địa phương gồm Hà Nội, Bắc Giang, Hà Giang. Hà Nội là thị trường tiêu thụ thực phẩm lớn, các sản phẩm thực phẩm rất phong phú, đa dạng. Trong khi, Hà Giang là một tỉnh phía Bắc có thói quen tích trữ thực phẩm sau thu hoạch, bảo quản thực phẩm tại các hộ kinh doanh nhỏ lẻ chưa tốt, dễ gây nấm mốc. Mẫu kiểm soát chất lượng được chuẩn bị bằng cách thêm chuẩn vào mẫu trắng tại mức $M L$ của các chất và phân tích cùng các lô mẫu phân tích. Kết quả phân tích mẫu được trình bày trong Bảng 5 . 
Bảng 5. Kết quả phân tích độc tố vi nấm trong các mẫu thực phẩm

\begin{tabular}{|c|c|c|c|c|c|c|}
\hline Loại mẫu & $\begin{array}{c}\text { Số } \\
m \text { mâu }\end{array}$ & Mycotoxin & $\begin{array}{l}\text { Số mẫu bi } \\
\text { nhiễm }\end{array}$ & $\begin{array}{c}\text { Tỷ lẹ bi } \\
\text { nhiếm (\%) }\end{array}$ & $\begin{array}{c}\text { Trung bình } \\
\text { các mẫu bị } \\
\text { nhiếm }(\mu g / k g)\end{array}$ & $\begin{array}{c}\text { Khoảng } \\
\text { nồng độ } \\
\text { bị nhiếm } \\
(\mu g / k g)\end{array}$ \\
\hline \multirow[t]{2}{*}{$\begin{array}{c}\text { Bánh/ } \\
\text { kẹo các loại }\end{array}$} & 40 & Aflatoxin & 1 & 2,5 & 5,7 & 5,7 \\
\hline & & Aflatoxin & 10 & 25 & 30 & $5,2-78$ \\
\hline \multirow[t]{3}{*}{ Gia vị các loại } & 40 & Ochratoxin A & 2 & 5,0 & 31 & $13-48$ \\
\hline & & Zearalenone & 2 & 5,0 & 7,0 & $5,8-8,2$ \\
\hline & & Aflatoxin & 10 & 17 & 6,4 & $0,7-22$ \\
\hline \multirow[t]{2}{*}{$\begin{array}{c}\text { Ngũ cốc } \\
\text { nguyên liệu }\end{array}$} & 60 & Ochratoxin A & 2 & 3,3 & 15 & $7,6-22$ \\
\hline & & Zearalenone & 1 & 1,7 & 56 & 56 \\
\hline \multirow{2}{*}{$\begin{array}{l}\text { Thực phẩm } \\
\text { bảo vệ sức } \\
\text { khỏe nguồn } \\
\text { gốc thảo dược }\end{array}$} & & Aflatoxin & 1 & 1,7 & 1,1 & 1,1 \\
\hline & 40 & Ochratoxin A & 1 & 1,7 & 46 & 46 \\
\hline \multirow{2}{*}{$\begin{array}{l}\text { Bột ngũ cốc } \\
\text { dinh duoõng }\end{array}$} & 60 & Aflatoxin & 7 & 12 & 1,9 & $1,1-3,3$ \\
\hline & 00 & Zearalenone & 1 & 1,7 & 32 & 32 \\
\hline Bột thính trộn & 60 & Aflatoxin & 5 & 8,3 & 1,8 & $1,6-1,9$ \\
\hline
\end{tabular}

Các aflatoxin bị phát hiện trong các mẫu với tỷ lệ cao hơn cả. Các kết quả cho thấy nhóm mẫu có tỷ lệ dương tính cao nhất là nhóm gia vị (chủ yếu là ớt bột), nhóm ngũ cốc nguyên liệu, chiếm tỷ lệ là $25 \%$. Có 07 mẫu ớt bột, 02 mẫu cari nghệ, 03 mẫu bột điều, 01 mẫu sa tế, 01 mẫu hạt tiêu phát hiện mycotoxin. Độc tố vi nấm được phát hiện nhiêu nhất là aflatoxin, một số phát hiện ochratoxin $\mathrm{A}$ và zearalenone. Một số mẫu phát hiện đông thời aflatoxin và ochratoxin $\mathrm{A}$ hoặc aflatoxin và zearalenone. Việc nhiễm đông thời nhiều loại độc tố vi nấm có thể gây độc do tương tác đông thời của các mycotoxin, ảnh hưởng đến sức khỏe người tiêu dùng.

Nhóm mẫu ngũ cốc nguyên liệu phát hiện 11 mẫu nhiễm độc tố vi nấm, chiếm tỷ lệ 16,7\%, trong đó có 02 mẫu lạc, 07 mẫu vừng, 01 mẫu gạo, 02 mẫu đỗ xanh. Số mẫu nhiễm mycotoxin vượt mức ML là 04 mẫu. Phát hiện 01 mẫu gạo nhiễm ochratoxin $\mathrm{A}$ ở mức $7,6 \mu \mathrm{g} / \mathrm{kg}$, vượt mức giới hạn cho phép theo QCVN 8-1:2011/BYT là $5 \mu \mathrm{g} / \mathrm{kg}$. Tỷ lệ nhiễm độc tố vi nấm ở nhóm ngũ cốc nguyên liệu là 6,7\%, giảm đáng kể so với số liệu thống kê năm 2017 [9] khảo sát tại Hà Giang và Bắc Giang.

Nhóm thực phẩm bảo vệ sức khỏe chỉ phát hiện 2 mẫu nhiễm mycotoxin, chiếm tỷ lệ $5 \%$. Tuy nhiên có 01 mẫu nhiễm ochratoxin A ở mức cao, $46 \mu \mathrm{g} / \mathrm{kg}$. Hiện chưa có quy định giới hạn tối đa cho OTA, tuy nhiên đây là mức dương tính cao so với các đối tượng thực phẩm thông thường khác, cỡ 0,5 - $30 \mu \mathrm{g} / \mathrm{kg}$ theo QCVN 8-1:2011/BYT. 
Nhóm bánh kẹo có một mẫu nhiễm mycotoxin và vượt ngưỡng cho phép của Bộ $\mathrm{Y}$ tế. Nhóm bột ngũ cốc dinh dưỡng và bột thính trộn phát hiện một số mẫu dương tính với mycotoxin, chiếm tỷ lệ lần lượt là $11,7 \%$ và $8,3 \%$, không có mẫu nào vượt giới hạn cho phép. Một số độc tố vi nấm không phát hiện trong các mẫu được khảo sát, bao gồm: deoxynivalenol, fumonisin B1.

Trong số 43 mẫu nhiễm độc tố vi nấm được khảo sát, có 17 mẫu phát hiện độc tố vi nấm vượt giới hạn cho phép theo quy định QCVN 8-1 : 2011/BYT, chiếm tỷ lệ 5\% tổng số mẫu được lấy (Hình 3).

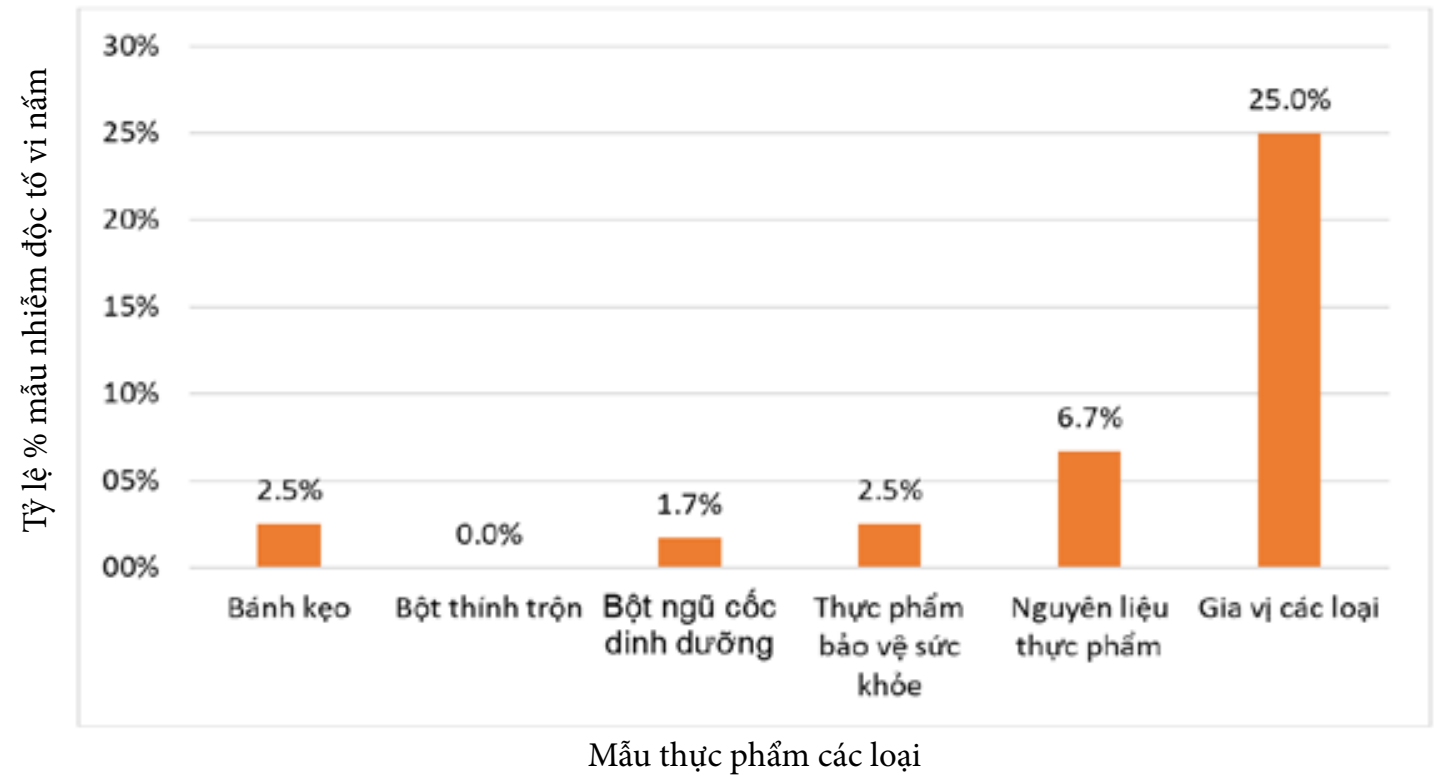

Hinh 3. Tỷ lẹ (\%) mẫu nhiễm độc tố vi nấm vượt mức quy định theo QCVN 8-1:2011/BYT

Nhóm mẫu gia vị là nhóm có tỷ lệ nhiễm mycotoxin cao nhất trong đó có 10 mẫu chứa mycotoxin vượt ML. Hàm lượng mycotoxin trong các mẫu dao động trong khoảng từ $9-78 \mu \mathrm{g} /$ $\mathrm{kg}$, trong khi mức tối đa cho phép của aflatoxin B1 là $5 \mu \mathrm{g} / \mathrm{kg}$, của aflatoxin tổng là $10 \mu \mathrm{g} / \mathrm{kg}$. Mẫu thực phẩm lấy tại Hà Nội có tỷ lệ nhiễm mycotoxin cao nhất ở nhóm gia vị và ngũ cốc chưa qua chế biến. Các mẫu này hầu hết là các mẫu không có nguồn gốc rõ ràng, được thu mua ở các chợ truyền thồng trên địa bàn thành phố Hà Nội. Tỷ lệ số mẫu nhiễm mycotoxin ở các tỉnh Hà Nội, Hà Giang, Bắc Giang lần lượt là 14,7, 12, 16\% (Bảng 6).

Bảng 6. Tỷ lệ mẫu nhiếm mycotoxin ở các tỉnh

\begin{tabular}{cccc}
\hline Đối tượng mẫu & Hà Nội & Hà Giang & Bắc Giang \\
\hline Bánh/kẹo & $0(0 \%)$ & $0(0 \%)$ & $1(10 \%)$ \\
Bột thính trộn & $5(16,7 \%)$ & $0(0 \%)$ & $0(0 \%)$ \\
Bột ngũ cốc dinh duỗng & $1(3,3 \%)$ & $1(6,7 \%)$ & $6(40 \%)$ \\
Thụ̂c phâm bảo vệ súc khỏe & $0(0 \%)$ & $0(0 \%)$ & $2(20 \%)$ \\
Nguyên liệu thụ̂c phẩm & $9(30 \%)$ & $1(6,7 \%)$ & $2(13,3 \%)$ \\
Gia vị các loại & $7(35 \%)$ & $7(70 \%)$ & $1(10 \%)$ \\
Tống số & $\mathbf{2 2 ( 1 4 , 7 \% )}$ & $\mathbf{9 ( 1 2 \% )}$ & $\mathbf{1 2 ( 1 6 \% )}$ \\
\hline
\end{tabular}




\section{KẾT LUẬN}

Quy trình phân tích đông thời các độc tố vi nấm bằng sắc ký lỏng khối phổ hai lần đã được áp dụng để xác định 05 nhóm độc tố vi nấm trong 300 mẫu thực phẩm các loại. Tỷ lệ mẫu nhiễm mycotoxin vượt giới hạn tối đa cho phép khoảng $5 \%$, chủ yếu tập trung vào nhóm gia vị và nguyên liệu ngũ cốc chưa qua chế biến. Các độc tố vi nấm bị phát hiện vượt quá giới hạn cho phép là aflatoxin, ochratoxin $\mathrm{A}$. Các mẫu nhiễm mycotoxin vượt quy định gồm: 01 mẫu bánh kẹo, 01 mẫu bột ngũ cốc dinh dưỡng, 04 mẫu nguyên liệu thực phẩm, 10 mẫu gia vị. Ngoài ra, độc tố ochratoxin $\mathrm{A}$ đã được phát hiện trong nhóm thực phẩm bảo vệ sức khỏe ở mức hàm lượng cao, $46 \mu \mathrm{g} / \mathrm{kg}$, mặc dù hiện chưa có quy định giới hạn tối đa cho loại thực phẩm này. Nhóm nghiên cứu đề xuất tiếp tục giám sát tình trạng nhiễm mycotoxin ở các đối tượng này trong các năm tiếp theo để có số liệu đánh giá đầy đủ hơn.

\section{TÀI LIỆU THAM KHẢO}

[1]. L. Anfossi, C. Giovannoli and C. Baggiani, "Mycotoxin detection", Current Opinion in Biotechnology, vol. 37, pp. 120-126, 2016.

[2]. Á. M. Francisco, M. Valle-Algarra, R. Mateo, J. V. Gimeno-Adelantado, F. Mateo and M. Jiménez, "Survey of the mycobiota of Spanish malting barley and evaluation of the mycotoxin producing potential of species of Alternaria, Aspergillus and Fusarium", International Journal of Food Microbiology, vol 108, no. 2, pp. 196-203, 2006.

[3]. M. Sharman, J. Gilbert and J. Chelkowski, "A survey of the occurrence of the mycotoxin moniliformin in cereal samples from sources worldwide", Food Additives \& Contaminants, vol 8, no. 4, pp. 459-466, 1991.

[4]. G. Jard, T. Liboz, F. Mathieu, A. Guyonvarc'h and A Lebrihi, "Review of mycotoxin reduction in food and feed: from prevention in the field to detoxification by adsorption or transformation", Food Additives \& Contaminants: Part A, vol. 28, no. 11, pp. 1590-1609, 2011.

[5]. Y. Ren, Y. Zhang, S. Shao, Z. Cai, L. Feng, H. Pan and Z. Wang, "Simultaneous determination of multi-component mycotoxin contaminants in foods and feeds by ultra-performance liquid chromatography tandem mass spectrometry", Journal of Chromatography A, vol. 1143, no. 1-2, pp. 48-64, 2007.

[6]. C. Tabuc, I. Taranu and L. Calin, "Survey of mould and mycotoxin contamination of cereals in South-Eastern Romania in 2008-2010", Archiva Zootechnica, vol. 14, no. 4, pp. 25-38, 2011.

[7]. J. D. Di Mavungu, S. Monbaliu, M-L. Scippo, G. Maghuin-Rogister, Y-J. Schneider, Y. Larondelle, A. Callebaut, J. Robbens, C. Van Peteghem and S. De Saeger, "LC-MS/MS multi-analyte method for mycotoxin determination in food supplements", Food Additives and Contaminants, vol. 26, no. 6, pp. 885-895, 2009.

[8]. M. Sulyok, R. Krska and R. Schuhmacher, "Application of an LC-MS/MS based multi-mycotoxin method for the semi-quantitative determination of mycotoxins occurring in different types of food infected by moulds", Food Chemistry, vol. 119, no. 1, pp. 408-416, 2010.

[9]. D. H. Tuan, T. C. Son, L. D. Chi, N. T. H. Binh, L. T. P. Thảo, L. T. H. Hao, L. D. Tuyen, T. N. H. Thu, "Dietary exposure and health risk characterization of aflatoxin B1, ochratoxin A, fumonisin B1, and zearalenone in food from different provinces in Northern Vietnam", Food Control, vol. 112, 107108, 2020. 


\title{
The occurrence of mycotoxins in food collected from some northern provinces of Vietnam in 2019
}

\author{
Nguyen Thi Ha Binh, Nguyen Ngoc Son, Nguyen Thi Lan, Tran Cao Son \\ National Institute for Food Control, Hanoi
}

\section{Abstract}

This study investigated the content of aflatoxin, ochratoxin A, zearalenone, deoxynivalenol and fumonisin B1 in some food product. Samples were extracted and cleaned by QUEChERS approach. The extract was measured by liquid chromatography tandem mass spectrometry. The positive samples were reconfirmed by standard methods using immunoaffinity extraction. The detection limit of the method for aflatoxin, fumonisin B1, ochratoxin A, deoxynivalenol, zearalenone were $0.5,30,0.5,60$, and $3.0 \mu \mathrm{g} / \mathrm{kg}$, respectively. The recoveries of all analytes were in the range of $70-110 \%$. The repeatability coefficient of variation were in the range of $5,1-13 \%$. Among the 300 food samples tested, there were 43 samples detected with studied mycotoxins, accounting for $14.3 \%$, mainly focusing on unprocessed cereals. In which, 10 spice samples, 04 peanuts and sesame samples, 01 candy sample, 01 sample of nutritious cereal flour, and 01 food supplement sample were found to be contaminated with aflatoxin, ochratoxin A exceeding the maximum level of Ministry Of Health according to QCVN 8-1 : 2011/BYT.

Keywords: LC-MS/MS, aflatoxin, ochratoxin A, deoxynivalenol, zearalenone, fumonisin B1. 